\title{
Corrigendum to "Asymptotic prime divisors over complete intersection rings” [Math. Proc. Camb. Phil. Soc. 160 (3) (2016) 423-436]
}

\author{
BY DIPANKAR GHOSH AND TONY J. PUTHENPURAKAL \\ Department of Mathematics, \\ Indian Institute of Technology Bombay, \\ Powai, Mumbai 400076, India. \\ e-mails: dipankar@math.itb.ac.in; \\ tputhen@math.itb.ac.in
}

(Received 4 January 2017; Revised 24 May 2017)

\section{Abstract}

There was a gap in the proof of Theorem $4 \cdot 1$ of [1]. In this corrigendum, we correct the error.

\section{Introduction}

Set-up 1.1. Let $Q$ be a Noetherian ring of finite Krull dimension. Let $\mathbf{f}=f_{1}, \ldots, f_{c}$ be a $Q$-regular sequence. Set $A:=Q /(\mathbf{f})$. Suppose $M$ and $N$ are finitely generated $A$-modules, where $\operatorname{projdim}_{Q}(M)$ is finite. Let $I$ be an ideal of $A$.

In [1, theorem 3.1], we proved that $\bigcup_{n, i \geqslant 0} \operatorname{Ass}_{A}\left(\operatorname{Ext}_{A}^{i}\left(M, N / I^{n} N\right)\right)$ is a finite set. Complementing this finiteness result, in [1, theorem 4.1], we showed the following asymptotic stability: There exist $n_{0}, i_{0} \geqslant 0$ such that for all $n \geqslant n_{0}$ and $i \geqslant i_{0}$,

$$
\begin{aligned}
\operatorname{Ass}_{A}\left(\operatorname{Ext}_{A}^{2 i}\left(M, N / I^{n} N\right)\right) & =\operatorname{Ass}_{A}\left(\operatorname{Ext}_{A}^{2 i_{0}}\left(M, N / I^{n_{0}} N\right)\right), \\
\operatorname{Ass}_{A}\left(\operatorname{Ext}_{A}^{2 i+1}\left(M, N / I^{n} N\right)\right) & =\operatorname{Ass}_{A}\left(\operatorname{Ext}_{A}^{2 i_{0}+1}\left(M, N / I^{n_{0}} N\right)\right) .
\end{aligned}
$$

1.2. Our strategy to prove $[\mathbf{1}$, theorem $4 \cdot 1]$ is as follows:

(i) choose $\mathfrak{p} \in \bigcup_{n, i \geqslant 0} \operatorname{Ass}_{A}\left(\operatorname{Ext}_{A}^{i}\left(M, N / I^{n} N\right)\right)$;

(ii) for every fixed $l=0,1$, show that there exist $n_{l}, i_{l} \geqslant 0$ such that

$$
\begin{array}{rll}
\text { either } & \mathfrak{p} \in \operatorname{Ass}_{A}\left(\operatorname{Ext}_{A}^{2 i+l}\left(M, N / I^{n} N\right)\right) & \text { for all } n \geqslant n_{l} \text { and } i \geqslant i_{l} \\
\text { or } & \mathfrak{p} \notin \operatorname{Ass}_{A}\left(\operatorname{Ext}_{A}^{2 i+l}\left(M, N / I^{n} N\right)\right) & \text { for all } n \geqslant n_{l} \text { and } i \geqslant i_{l} .
\end{array}
$$

Localising at $\mathfrak{p}$, and replacing $A_{\mathfrak{p}}$ by $A$ and $\mathfrak{p} A_{\mathfrak{p}}$ by $\mathfrak{m}$, we may assume that $A$ is a local ring with maximal ideal $\mathfrak{m}$ and residue field $k$. In [1, lemma 4.2], we proved that the lengths $\lambda\left(\operatorname{Hom}_{A}\left(k, \operatorname{Ext}_{A}^{2 i}\left(M, N / I^{n} N\right)\right)\right)$ and $\lambda\left(\operatorname{Hom}_{A}\left(k, \operatorname{Ext}_{A}^{2 i+1}\left(M, N / I^{n} N\right)\right)\right)$ are given by polynomials in $n, i$ with rational coefficients for all sufficiently large $n, i$. Using this, we erroneously concluded the fact 1.2(ii). Our assertion would have been correct if $\bigoplus_{n, i \geqslant 0} \operatorname{Hom}_{A}\left(k, \operatorname{Ext}_{A}^{i}\left(M, N / I^{n} N\right)\right)$ is a finitely generated module over some appropriate Noetherian bigraded ring. However, we believe that this module is practically never finitely generated over the ring $\mathscr{S}=\mathscr{R}(I)\left[t_{1}, \ldots, t_{c}\right]$ we worked with (see [1, section 2]). In this corrigendum, we correct our oversight. We prove the following: 
LEMMA 1·3. Along with Set-up 1·1, further assume that $Q$ is a local ring with the residue field $k$. Then, for every fixed $l=0,1$, we have that:

$$
\begin{aligned}
\text { either } & \operatorname{Hom}_{A}\left(k, \operatorname{Ext}_{A}^{2 i+l}\left(M, N / I^{n} N\right)\right) \neq 0 \text { for all } n, i \gg 0 \\
\text { or } & \operatorname{Hom}_{A}\left(k, \operatorname{Ext}_{A}^{2 i+l}\left(M, N / I^{n} N\right)\right)=0 \text { for all } n, i \gg 0 .
\end{aligned}
$$

Using Lemma $1 \cdot 3$, one can easily prove the fact $1 \cdot 2$ (ii), and hence [1, theorem $4 \cdot 1]$.

\section{Proof of Lemma $1 \cdot 3$}

With Set-up 1.1, further assume that $\mathcal{N}=\bigoplus_{n \geqslant 0} N_{n}$ is a graded module over the Rees ring $\mathscr{R}(I):=\bigoplus_{n \geqslant 0} I^{n} X^{n}$. Then $\mathscr{E}(\mathcal{N}):=\bigoplus_{n, i \geqslant 0} \operatorname{Ext}_{A}^{i}\left(M, N_{n}\right)$ turns into a bigraded module over $\mathscr{S}:=\mathscr{R}(I)\left[t_{1}, \ldots, t_{c}\right]$, where $t_{j}: \operatorname{Ext}_{A}^{i}\left(M, N_{n}\right) \rightarrow \operatorname{Ext}_{A}^{i+2}\left(M, N_{n}\right), i \geqslant 0$, are the Eisenbud operators, and we set $\operatorname{deg}\left(t_{j}\right)=(0,2)$ for all $1 \leqslant j \leqslant c$ and $\operatorname{deg}\left(u X^{s}\right)=(s, 0)$ for all $u \in I^{s}, s \geqslant 0$; see [1, section 2.3]. Since $\mathcal{L}:=\bigoplus_{n \geqslant 0}\left(I^{n} N / I^{n+1} N\right)$ and $\mathcal{L}^{\prime}:=$ $\bigoplus_{n \geqslant 0}\left(N / I^{n} N\right)$ are graded $\mathscr{R}(I)$-modules, we obtain that

$$
\begin{aligned}
& U=\bigoplus_{n, i \geqslant 0} U_{(n, i)}:=\mathscr{E}(\mathcal{L})=\bigoplus_{n, i \geqslant 0} \operatorname{Ext}_{A}^{i}\left(M, I^{n} N / I^{n+1} N\right), \\
& V=\bigoplus_{n, i \geqslant 0} V_{(n, i)}:=\mathscr{E}\left(\mathcal{L}^{\prime}\right)=\bigoplus_{n, i \geqslant 0} \operatorname{Ext}_{A}^{i}\left(M, N / I^{n} N\right)
\end{aligned}
$$

are bigraded modules over $\mathscr{S}=\mathscr{R}(I)\left[t_{1}, \ldots, t_{c}\right]$. To prove Lemma 1.3, we use:

LEMMA 2.1. Let $A$ be a Noetherian ring and I an ideal of $A$. Let $\mathscr{R}(I)$ be the Rees ring of $I$. Set $\mathscr{S}:=\mathscr{R}(I)\left[t_{1}, \ldots, t_{c}\right]$, where $\operatorname{deg}\left(t_{j}\right)=(0,2)$ for all $1 \leqslant j \leqslant c$ and $\operatorname{deg}\left(I^{s}\right)=(s, 0)$ for all $s \geqslant 0$. Suppose $L=\bigoplus_{(n, i) \in \mathbb{N}^{2}} L_{(n, i)}$ is a finitely generated bigraded $\mathscr{S}$-module. Then, for every fixed $l=0,1$, we have that either $L_{(n, 2 i+l)} \neq 0$ for all $n, i \gg 0$; or $L_{(n, 2 i+l)}=0$ for all $n, i \gg 0$.

Proof. By virtue of $\left[3\right.$, proposition 5.1], there is $\left(n_{0}, i_{0}\right) \in \mathbb{N}^{2}$ such that

$$
\begin{aligned}
\operatorname{Ass}_{A}\left(L_{(n, 2 i)}\right) & =\operatorname{Ass}_{A}\left(L_{\left(n_{0}, 2 i_{0}\right)}\right) \quad \text { for all }(n, i) \geqslant\left(n_{0}, i_{0}\right) ; \\
\operatorname{Ass}_{A}\left(L_{(n, 2 i+1)}\right) & =\operatorname{Ass}_{A}\left(L_{\left(n_{0}, 2 i_{0}+1\right)}\right) \text { for all }(n, i) \geqslant\left(n_{0}, i_{0}\right) .
\end{aligned}
$$

The result now follows from the well-known fact: for an $A$-module $M, \operatorname{Ass}_{A}(M)$ is nonempty if and only if $M \neq 0$.

We now give:

Proof of Lemma 1.3. We prove the lemma for $l=0$ only. For $l=1$, the proof is similar. Set $f(n, i):=\lambda\left(\operatorname{Hom}_{A}\left(k, \operatorname{Ext}_{A}^{2 i}\left(M, N / I^{n} N\right)\right)\right)$ for all $n, i \geqslant 0$. By virtue of [1, lemma 4.2], $f(n, i)$ is given by a polynomial in $n, i$ with rational coefficients for all $n, i \gg 0$. If $f(n, i)=0$ for all $n, i \gg 0$, then there is nothing to prove. Suppose this is not the case. Then we claim that $\operatorname{Hom}_{A}\left(k, \operatorname{Ext}_{A}^{2 i}\left(M, N / I^{n} N\right)\right) \neq 0$ for all $n, i \gg 0$.

For every $n \geqslant 0$, the exact sequence $0 \rightarrow I^{n} N / I^{n+1} N \rightarrow N / I^{n+1} N \rightarrow N / I^{n} N \rightarrow 0$ yields an exact sequence of $A$-modules (for each $i$ ):

$$
\begin{aligned}
& \operatorname{Ext}_{A}^{i}\left(M, I^{n} N / I^{n+1} N\right) \longrightarrow \operatorname{Ext}_{A}^{i}\left(M, N / I^{n+1} N\right) \longrightarrow \operatorname{Ext}_{A}^{i}\left(M, N / I^{n} N\right) \\
& \longrightarrow \operatorname{Ext}_{A}^{i+1}\left(M, I^{n} N / I^{n+1} N\right) .
\end{aligned}
$$


Taking direct sum over $n, i$, and using the naturality of the Eisenbud operators $t_{j}$, we have an exact sequence $U \stackrel{\Phi}{\longrightarrow} V(1,0) \stackrel{\Xi}{\longrightarrow} V \stackrel{\Psi}{\longrightarrow} U(0,1)$ of bigraded modules over $\mathscr{S}=$ $\mathscr{R}(I)\left[t_{1}, \ldots, t_{c}\right]$, where $U$ and $V$ are as in $(2 \cdot 1 a)$ and $(2 \cdot 1 b)$ respectively. Hence, setting $X:=$ Image $(\Phi), Y:=$ Image $(\Xi)$ and $Z:=$ Image $(\Psi)$, we obtain the short exact sequences: $0 \rightarrow X \rightarrow V(1,0) \rightarrow Y \rightarrow 0$ and $0 \rightarrow Y \rightarrow V \rightarrow Z \rightarrow 0$. Applying $\operatorname{Hom}_{A}(k,-)$ to these short exact sequences, we get the following exact sequences:

$$
\begin{gathered}
0 \longrightarrow \operatorname{Hom}_{A}(k, X) \longrightarrow \operatorname{Hom}_{A}(k, V(1,0)) \longrightarrow \operatorname{Hom}_{A}(k, Y) \longrightarrow C \longrightarrow 0, \\
0 \longrightarrow \operatorname{Hom}_{A}(k, Y) \longrightarrow \operatorname{Hom}_{A}(k, V) \longrightarrow D \longrightarrow 0,
\end{gathered}
$$

where $C:=\operatorname{Image}\left(\operatorname{Hom}_{A}(k, Y) \rightarrow \operatorname{Ext}_{A}^{1}(k, X)\right)$ and $D:=\operatorname{Image}\left(\operatorname{Hom}_{A}(k, V) \rightarrow\right.$ $\left.\operatorname{Hom}_{A}(k, Z)\right)$. By virtue of [2, theorem 1.1], $U$ is a finitely generated bigraded $\mathscr{S}$-module, and hence $X$ and $Z$ are so. This implies that $\operatorname{Hom}_{A}(k, X), \operatorname{Ext}_{A}^{1}(k, X)$ and $\operatorname{Hom}_{A}(k, Z)$ are finitely generated bigraded $\mathscr{S}$-modules. Therefore $C$ and $D$ are finitely generated bigraded $\mathscr{S}=\mathscr{R}(I)\left[t_{1}, \ldots, t_{c}\right]$-modules. Hence, by Lemma $2 \cdot 1$, we get:

$$
\left\{\begin{aligned}
\text { either } & \operatorname{Hom}_{A}\left(k, X_{(n, 2 i)}\right) \neq 0 \text { for all } n, i \gg 0 \\
\text { or } & \operatorname{Hom}_{A}\left(k, X_{(n, 2 i)}\right)=0 \text { for all } n, i \gg 0
\end{aligned}\right\}
$$

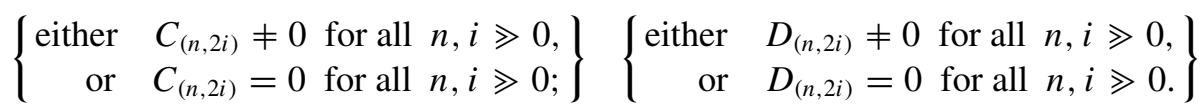

For $n, i \geqslant 0$, the $(n, 2 i)$ th components of $(2 \cdot 2 a)$ and $(2 \cdot 2 b)$ yield the exact sequences:

$0 \longrightarrow \operatorname{Hom}_{A}\left(k, X_{(n, 2 i)}\right) \longrightarrow \operatorname{Hom}_{A}\left(k, V_{(n+1,2 i)}\right) \longrightarrow \operatorname{Hom}_{A}\left(k, Y_{(n, 2 i)}\right) \longrightarrow C_{(n, 2 i)} \longrightarrow 0$,

$$
0 \longrightarrow \operatorname{Hom}_{A}\left(k, Y_{(n, 2 i)}\right) \longrightarrow \operatorname{Hom}_{A}\left(k, V_{(n, 2 i)}\right) \longrightarrow D_{(n, 2 i)} \longrightarrow 0 .
$$

Now we are in a position to prove our claim that $\operatorname{Hom}_{A}\left(k, V_{(n, 2 i)}\right) \neq 0$ for all $n, i \gg 0$. We consider the following four cases:

Case 1. Assume that $\operatorname{Hom}_{A}\left(k, X_{(n, 2 i)}\right) \neq 0$ for all $n, i \gg 0$. Then, in view of $(2.4 a)$, we get that $\operatorname{Hom}_{A}\left(k, V_{(n, 2 i)}\right) \neq 0$ for all $n, i \gg 0$. So, in this case, we are done.

Case 2. Assume that $C_{(n, 2 i)} \neq 0$ for all $n, i \gg 0$. So again, in view of $(2.4 a)$, we have that $\operatorname{Hom}_{A}\left(k, Y_{(n, 2 i)}\right) \neq 0$ for all $n, i \gg 0$. Hence $(2.4 b)$ yields that $\operatorname{Hom}_{A}\left(k, V_{(n, 2 i)}\right) \neq 0$ for all $n, i \gg 0$. Thus, in this case also, we are done.

Case 3. Assume that $D_{(n, 2 i)} \neq 0$ for all $n, i \gg 0$. In this case, $(2 \cdot 4 b)$ gives that $\operatorname{Hom}_{A}\left(k, V_{(n, 2 i)}\right) \neq 0$ for all $n, i \gg 0$, and hence we are done.

In view of (2.3), if none of the above three cases holds, then we have the following:

Case 4. Assume that $\operatorname{Hom}_{A}\left(k, X_{(n, 2 i)}\right)=0$ for all $n, i \gg 0, C_{(n, 2 i)}=0$ for all $n, i \gg 0$, and $D_{(n, 2 i)}=0$ for all $n, i \gg 0$. Hence the exact sequences $(2.4 a)$ and $(2.4 b)$ yield the isomorphisms: $\operatorname{Hom}_{A}\left(k, V_{(n+1,2 i)}\right) \cong \operatorname{Hom}_{A}\left(k, Y_{(n, 2 i)}\right) \cong \operatorname{Hom}_{A}\left(k, V_{(n, 2 i)}\right)$ for all $n, i \gg 0$. These isomorphisms provide the following equalities:

$$
f(n+1, i)=f(n, i) \text { for all } n, i \gg 0 .
$$

We may write the polynomial expression of $f(n, i)$ in the following way:

$$
f(n, i)=h_{0}(i) n^{a}+h_{1}(i) n^{a-1}+\cdots+h_{a-1}(i) n+h_{a}(i) \text { for all } n, i \gg 0,
$$

where $h_{j}(i), 0 \leqslant j \leqslant a$, are polynomials in $i$ over $\mathbb{Q}$. We may assume without loss of generality that $h_{0}$ is a non-zero polynomial. Therefore $h_{0}$ may have only finitely many roots. 
Let $i^{\prime} \geqslant 0$ be such that $h_{0}(i) \neq 0$ for all $i \geqslant i^{\prime}$. In view of (2.5) and (2.6), there exist some $n_{0}(\geqslant 0)$ and $i_{0}\left(\geqslant i^{\prime}\right.$, say) such that for all $n \geqslant n_{0}$ and $i \geqslant i_{0}$, we have

$$
f(n+1, i)=f(n, i) \quad \text { and } \quad f(n, i)=h_{0}(i) n^{a}+h_{1}(i) n^{a-1}+\cdots+h_{a-1}(i) n+h_{a}(i) .
$$

These equalities imply that $a$ must be equal to 0 , and hence $f(n, i)=h_{0}(i)$ for all $n \geqslant n_{0}$ and $i \geqslant i_{0}$. Thus $f(n, i) \neq 0$ for all $n \geqslant n_{0}$ and $i \geqslant i_{0}$, and hence $\operatorname{Hom}_{A}\left(k, V_{(n, 2 i)}\right) \neq 0$ for all $n \geqslant n_{0}$ and $i \geqslant i_{0}$, which completes the proof of Lemma 1.3.

Acknowledgments. We thank Prof. Vijaylaxmi Trivedi for showing us the gap in our paper.

\section{REFERENCES}

[1] D. Ghosh and Tony J. Puthenpurakal. Asymptotic prime divisors over complete intersection rings. Math. Proc. Camb. Phil. Soc. 160 (2016), 423-436.

[2] Tony J. Puthenpurakal. On the finite generation of a family of Ext modules. Pacific J. Math. 266 (2013), 367-389.

[3] E. WeST. Primes associated to multigraded modules. J. Algebra 271 (2004), 427-453. 